\title{
Synchronization of Multiple Time-of-Flight Cameras Using Photodiodes
}

2020 | Conference Paper | Accepted Manuscript (Postprint)

available at https://doi.org/10.18452/23672

Final version published as:

Thorben Wübbenhorst, Felix Wermke, Beate Meffert: Synchronization of Multiple Time-of-Flight Cameras Using Photodiodes. In: 2020 IEEE SENSORS. DOI:

10.1109/SENSORS47125.2020.9278774

(C) 2020 IEEE. Personal use of this material is permitted. Permission from IEEE must be obtained for all other uses, in any current or future media, including reprinting/republishing this material for advertising or promotional purposes, creating new collective works, for resale or redistribution to servers or lists, or reuse of any copyrighted component of this work in other works.

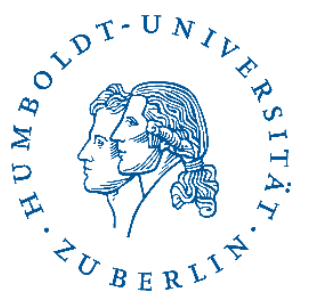

edoc-Server

Open-Access-Publikationsserver

der Humboldt-Universität zu Berlin 


\title{
Synchronization of Multiple Time-of-Flight Cameras Using Photodiodes
}

\author{
Thorben Wübbenhorst, Felix Wermke, Beate Meffert \\ Department of Computer Science \\ Humboldt-Universität zu Berlin \\ Berlin, Germany \\ wuebbent@informatik.hu-berlin.de
}

\begin{abstract}
Time-of-flight (ToF) cameras are an evolving technology, finding applications in a wide range of fields, including machine vision, automotive applications, face or hand recognition, and human-robot collaboration. A setup consisting of multiple ToF cameras can provide a 3-D model of the recorded scene with a larger field of view and avoid occlusion. The operating principle of ToF cameras' requires the emission of light to measure its round-trip time to the objects in the field of view and the reflection back to the camera. If multiple ToF cameras operate simultaneously, their light waves may interfere, resulting in erroneous depth measurements. In this paper, we propose a multi-camera time-of-flight system based on dynamic time-division multiple access (dynamic TDMA) to avoid any interference. The time synchronization is implemented optically, so that no further time synchronization, external to the cameras, is required. To achieve a high frame rate, every camera is extended with a photodiode, signaling a free channel. A synchronization protocol is implemented to allow for an autonomous operation of the ToF cameras.

Index Terms-Autonomous synchronization, multi-camera interference, multiple camera system, optical synchronization, timeof-flight camera.
\end{abstract}

\section{INTRODUCTION}

Time-of-Flight (ToF) cameras acquire depth images by emitting infrared light (illumination) and measuring the time between emission and the received reflection of objects in the scene. For many applications, e.g. industrial safety environments, it is necessary to use multiple cameras to reduce occlusion, shadowed objects or persons, which are unable to be seen from a particular field of view. [1], [2]. Multiple ToF cameras, using the same modulation frequency, may interfere with each other and cause errors in the distance measurements [3]-[8]. To avoid interference errors Büttgen and Seitz proposed channel access methods adopted from communication systems: space-division multiple access (SDMA), wavelength-division multiple access (WDMA), time-division multiple access (TDMA), frequency-division multiple access (FDMA) and code-division multiple access (CDMA) [9], [10]. Camera vendors commonly implement FDMA for multicamera systems. FDMA assigns every ToF camera a unique modulation frequency to reduce measurement errors due to interference [11]. The other cameras' modulated light is sensed

\footnotetext{
*This research was performed in cooperation with iris $\mathrm{GmbH}$ and is cofinanced by the European Regional Development Fund (ERDF)
}

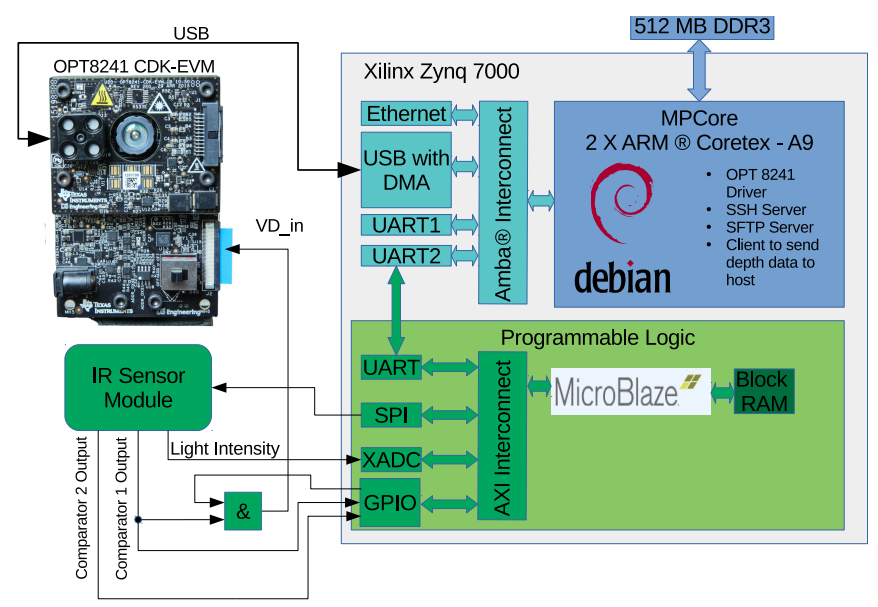

Fig. 1. System overview of one Time-of-Flight camera used in the experiments. The camera consists of a Texas Instruments OPT8241 developer kit connected to a Xilinx Zynq-7000 System-on-Chip, as well as an additional infrared (IR) sensor module, which is also connected to the Zynq.

as ambient light. With an increasing number of ToF cameras, the amount of ambient light increases leading to saturated pixels and consequently erroneous depth measurements. A naive solution would be to reduce the illumination power, but with reduced power the signal-to-noise ratio deteriorates, which in turn leads to a less precise distance measurement.

One possibility to avoid any interference is to use TDMA [12], [13]. The disadvantage of TDMA is the decreasing frame rate with an increasing number of cameras. For this reason, Wermke [13] proposes to use the readout time, the time between consecutive integration phases, for the other cameras to acquire their depth images. This leads to only a small reduction in the frame rate of the multi-camera system. However, the time slots have a constant duration, which, on the one hand, must be long enough to cover the longest possible integration time required, and on the other hand, is an inefficient use of time when only short integration times are used.

This work proposes a multi-camera operation based on time multiplexing to avoid interference. The time synchronization is performed optically by an additional photodiode connected to 
the ToF camera. The advantage of the optical synchronization is in the easy setup of the multi-camera system, which can synchronize itself. The use of the photodiode provides fast sensing capabilities to the cameras and are used to detect the end of another camera's image acquisition and to then start the own acquisition, thus implementing carrier-sense multiple access (CSMA). This makes it possible to arrange the cameras in consecutive order without static time slot lengths.

This paper is organized as follows. Section II presents the synchronization procedure, followed by Section III covering the maximum achievable frame rate. The implementation of the camera setup is presented in Section IV, the experimental setup with measurements and results in Section V. This paper closes with discussions in Section VI and conclusions in Section VII.

\section{SYNCHRONIZATION}

The ToF cameras operate in consecutive order, one after the other. The use of the photodiode allows for fast detection of other cameras' acquisition phases and is therefore used to start the own acquisition phase shortly after the previous camera's end of acquisition.

The first ToF camera acquires a depth image. This process is sensed by the second ToF camera, which starts its own depth image acquisition immediately after the end of the first. This continues until the last operating camera. The first camera then starts its acquisition phase after a period $t_{n o \_} c a m$, where no other camera's illumination is detected. This time $t_{n o \_c a m}$ is used to mark the end of one round of acquisitions and is known a priori by all cameras. Therefore, the time between two cameras' acquisitions $t_{\text {delay }}$ must be lower than $t_{n o \_}$cam. When a new camera is turned on, it detects the time interval with length $t_{n o \_}$cam and starts its acquisition after the last camera before the time $t_{n o \_}$cam has lapsed. Every camera has to count the number of operating cameras and remember its position in the sequence. The first camera will detect the additional camera and delay its next acquisition to allow for operation without interference.

\section{FRAME RATE}

The achievable frame rate mainly depends on the numbers of operating cameras $n$, their individual integration times $t_{i n t}$, and their readout times $t_{\text {readout }}$. The standard operation of an amplitude modulated continuous wave (AMCW) ToF camera is to acquire a depth image consisting of four frames. The duration of one depth image acquisition $t_{\text {cycle }}$ of all $n$ cameras is calculated with (1).

$t_{\text {cycle }}=t_{\text {no_cam }_{-}}+(n-1) \cdot t_{\text {delay }}+\sum_{i=1}^{n}\left(4 \cdot t_{\text {int }_{i}}+3 \cdot t_{\text {readout }_{i}}\right)$

The subscript $i$ indicates the parameters for camera $i$ out of the $n$ cameras. The time of the last readout of every image acquisition is already used by the next camera. The frame rate per camera is calculated as

$$
\text { frame_rate }=\frac{1}{t_{\text {cycle }}}
$$

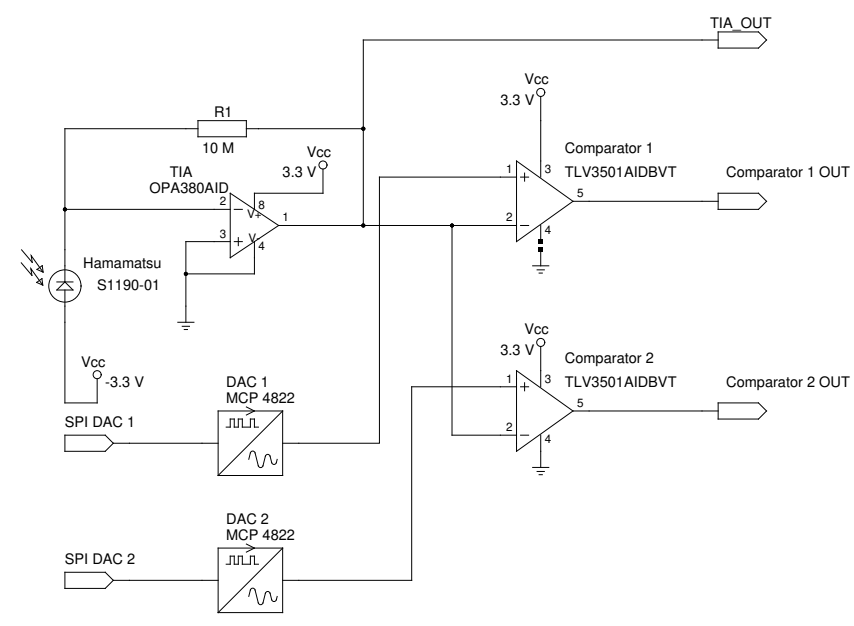

Fig. 2. Schematic of the sensor module.

\section{IMPLEMENTATION}

The proposed synchronization method requires ToF cameras with a shutter input, that can be triggered externally, so that the image acquisition can be started precisely from an external source. Examples of such cameras/sensors are the Espros EPC660, Melexis EVK75123, or the Texas Instruments OPT8241. In our setup, the evaluation kit from Texas Instruments OPT8241-CDK-EVM are used. This camera is connected to the USB port of a ZedBoard from Digilent Inc. Additionally, a sensor module containing the photodiode is also connected to the ZedBoard. The ZedBoard contains a Xilinx Zynq-7000 System on Chip, including a field-programmable gate array (FPGA) for programmable logic and an ARM processor. The ARM processor of the Zynq hosts a Debian Linux. The Linux operating system runs the driver software for the camera, to configure the ToF camera and to receive the depth images. The depth images are forwarded from the Zynq to a $\mathrm{PC}$ via ethernet for evaluation and display. The programmable logic of the Zynq contains a Microblaze softcore processor. This Microblaze is used to configure the sensor module and runs the synchronization software. FreeRTOS is used to satisfy the real-time requirement of the operation.

\section{A. Sensor}

Fig. 2 shows the schematic of the sensors module, which is connected to the ZedBoard. It contains the PIN photodiode (left), the comparators (right), the digital to analog converters (DAC) (bottom left), and the transimpedance amplifier (TIA) (top left). The PIN photodiode is connected to the transimpedance amplifier. The output of the amplifier is connected to two comparators. The switching thresholds of the comparators are set with two digital to analog converters (DAC), controlled by the Microblaze in the ZedBoard. The first comparator is used to detect the integration phases of the other cameras. The second comparator is used to sense other cameras operating simultaneously to the own camera. Each time the output of comparator 1 changes, the software reads the voltage level at the output of the TIA $U_{T I A}$ and sets the 
TABLE I

CAMERA PARAMETERS DURING THE EXPERIMENTS

\begin{tabular}{|c|c|c|}
\hline Camera & $t_{\text {int }}$ & $t_{\text {readout }}$ \\
\hline Camera 1 & $1.2 \mathrm{~ms}$ & $3 \mathrm{~ms}$ \\
Camera 2 & $0.2 \mathrm{~ms}$ & $3.98 \mathrm{~ms}$ \\
Camera 3 & $0.68 \mathrm{~ms}$ & $3.5 \mathrm{~ms}$ \\
\hline
\end{tabular}

threshold voltages of the comparators $U_{c o m p 1}$ and $U_{c o m p 2}$. If the ambient light changes, e.g. the sun is suddenly covered by clouds, the thresholds of the comparators are adapted automatically. The comparator thresholds are set as follows. If no illumination is received $U_{c o m p 1}=U_{T I A}+200 \mathrm{mV}$ and $U_{\text {comp } 2}=U_{T I A}+200 \mathrm{mV}$, otherwise $U_{c o m p 1}=U_{T I A}-$ $200 \mathrm{mV}$ and $U_{\text {comp } 2}=U_{T I A}+200 \mathrm{mV}$.

\section{B. Synchronized start of image acquisition}

The synchronization algorithm counts the number of acquired frames per camera. Once it is the camera's turn to acquire a depth image and the previous camera has acquired its third (out of four) frames, an 'enable' signal is activated.

The shutter signal of the ToF camera (VD_in in Fig. 1) is driven by an AND gate, which is connected to the synchronization algorithm's enable signal and the comparator output of the sensor module. As soon as the comparator signals that the current active illumination has been turned off, the acquisition process of the camera starts. That way, a fast reaction time, a small $t_{\text {delay }}$, is realized.

\section{Experimental Setup, Measurements and Results}

\section{A. Experimental setup}

In the experimental setup, three ToF cameras acquire depth images of the object from different perspectives. The fields of view of the ToF cameras overlap so that all cameras detect the light emitted by the other cameras.

The ToF camera chips OPT8241 are configured as follows:

- slave_mode: on

- frequency sweeping: off

- 4 frames and 1 sub-frame

- Modulation frequency: $60 \mathrm{MHz}$

Table I shows the individual integration and readout times for three ToF cameras in this setup.

\section{B. Measurements and results}

The first measurement was undertaken to determine the accuracy of the detection of illumination. This measurement revealed that the start of the illumination phase is detected after $610 \mathrm{~ns}$ by the sensor board and the end of illumination after $11 \mu$ s. These different times are inherent to the sensor structure. The variance of the detection of the end of illumination is shown in Fig. 3 as a histogram. The time between the actual end of the illumination and the detection by the sensor module was recorded. The offset to the end of illumination is $11 \mu \mathrm{s}$, the standard deviation of the detection is $374 \mathrm{~ns}$, and the time interval to cover all samples is $2.4 \mu \mathrm{s}$. The delay within the ToF camera chip from applying the shutter until the illumination is active is about $23 \mu \mathrm{s}$. Therefore, $t_{\text {delay }}$ is about $34 \mu \mathrm{s}$.

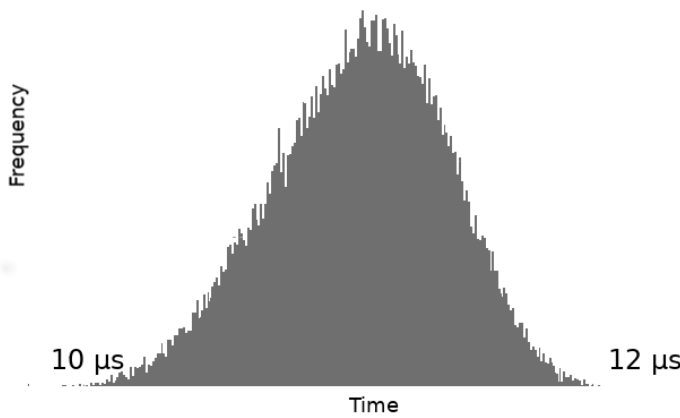

Fig. 3. The histogram shows the detection accuracy of the end of illumination. The time difference of the end of illumination of the first camera and the start of a frame of a second camera is displayed. The offset to the end of illumination is about $11 \mu \mathrm{s}$. The width of the histogram is $2.4 \mu \mathrm{s}$ with a standard deviation of $374 \mathrm{~ns}$.

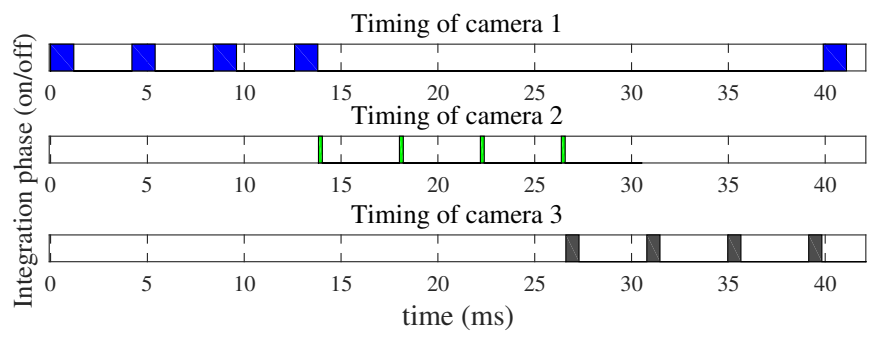

Fig. 4. The timing diagram of measurement two shows the consecutive depth image acquisitions of the three ToF cameras. The acquisitions repeats after $t_{\text {cycle }}=39.9 \mathrm{~ms}$. The bars represent the integration phases, in between are the readout phases.

The second measurement was conducted to test the functionality of the synchronization algorithm with three $\mathrm{ToF}$ cameras. The cameras acquired depth images in the described consecutive arrangement for three hours. Fig. 4 shows the timing behaviour of the cameras. To allow for some tolerance, $t_{n o \_}$cam was set to $70 \mu$ s. During this experiment, no interference was recorded. The integration and readout times of all cameras result in $t_{\text {cycle }}=39.9 \mathrm{~ms}$, which is about 25 depth images per second per camera. The achieved $t_{\text {delay }}$ and $t_{n o \_}$cam are very small compared to the overall acquisition times.

\section{DISCUSSION}

The results prove the initial hypothesis that an additional photodiode may be used to detect the illumination of other ToF cameras. Furthermore, the time to detect the end of illumination is in the range of $\mu \mathrm{s}$, allowing a fast reaction time to allow for a sequential camera operation.

\section{CONCLUSiOn}

In this work, a new approach for a multi-ToF-camera system is presented, which is based on TDMA to avoid interferencerelated errors. A photodiode, attached the every ToF camera, proved suitable to control the consecutive operation of the cameras. Furthermore, it allows for variable integration times of the ToF cameras and a higher frame rate than the standard implementation of TDMA using static time slot lengths. 


\section{REFERENCES}

[1] J. Mure-Dubois and H. Hügli, "Fusion of time of flight camera point clouds," in Workshop on Multi-camera and Multi-modal Sensor Fusion Algorithms and Applications - M2SFA2 2008. Marseille, France: Andrea Cavallaro and Hamid Aghajan, Oct. 2008. [Online]. Available: https://hal.inria.fr/inria-00326781

[2] M. Otto, P. Agethen, F. Geiselhart, and E. Rukzio, "Towards ubiquitous tracking: Presenting a scalable, markerless tracking approach using multiple depth cameras," in Proceedings of EuroVR Conference 2015, 2015, pp. 14-20.

[3] F. Wermke and B. Meffert, "Interference model of two time-of-flight cameras," in 2019 IEEE SENSORS, 2019.

[4] L. A. Seewald, V. F. Rodrigues, M. Ollenschläger, R. S. Antunes, C. A. da Costa, R. da Rosa Righi, L. G. da Silveira, A. Maier, B. Eskofier, and R. Fahrig, "Toward analyzing mutual interference on infrared-enabled depth cameras," Computer Vision and Image Understanding, vol. 178, pp. 1 - 15, 2019. [Online]. Available: http://www.sciencedirect.com/science/article/pii/S1077314218303400

[5] A. Kunz, L. Brogli, and A. Alavi, "Interference measurement of kinect for xbox one," in Proceedings of the 22Nd ACM Conference on Virtual Reality Software and Technology. ACM, 11 2016, pp. 345-346. [Online]. Available: http://doi.acm.org/10.1145/2993369.2996329

[6] S. Bauer, A. Seitel, H. G. Hofmann, T. Blum, J. Wasza, M. Balda, H.-P. Meinzer, N. Navab, J. Hornegger, and L. Maier-Hein, "Real-time range imaging in health care: A survey," in Time-of-Flight and Depth Imaging, 2013.
[7] J. Volak, D. Koniar, F. Jabloncik, L. Hargas, and S. Janisova, "Interference artifacts suppression in systems with multiple depth cameras," in 2019 42nd International Conference on Telecommunications and Signal Processing (TSP), 07 2019, pp. 472-476.

[8] Y. M. Kim, D. Chan, C. Theobalt, and S. Thrun, "Design and calibration of a multi-view tof sensor fusion system," in 2008 IEEE Computer Society Conference on Computer Vision and Pattern Recognition Workshops, June 2008, pp. 1-7.

[9] B. Büttgen, Extending Time-of-flight Optical 3D-imaging to Extreme Operating Conditions. Sierke, 2007. [Online]. Available: https://books.google.de/books?id=eItHNAAACAAJ

[10] B. Buttgen and P. Seitz, "Robust optical time-of-flight range imaging based on smart pixel structures," IEEE Transactions on Circuits and Systems I: Regular Papers, vol. 55, no. 6, pp. 1512-1525, July 2008.

[11] R. Lange, "3d time-of-flight distance measurement with custom solidstate image sensors in cmos/ccd-technology," Ph.D. dissertation, University of Siegen, Germany, 2000. [Online]. Available: http://dokumentix.ub.uni-siegen.de/opus/volltexte/2006/178

[12] T. Wübbenhorst, "Frame-basierte optische synchronisation von time-offlight (tof) sensoren," Master's thesis, Humboldt-Universität zu Berlin, 2019.

[13] F. Wermke, T.Wübbenhorst, and B. Meffert, "Interference avoidance for two time-of-flight cameras using autonomous optical synchronization," in 2020 6th International Conference on Control, Automation and Robotics (ICCAR), 2020, pp. 586 - 595. 